\title{
Low Effective Material Loss TOPAS Based Single- Mode Photonic Crystal Fiber with High Core Power Fraction in the THz Waveguiding
}

\section{Selim Hossain}

KYAU https://orcid.org/0000-0003-0715-4873

Shuvo Sen ( $\square$ shuvombstu.it12009@gmail.com )

Mawlana Bhashani Science and Technology University

\section{Research Article}

Keywords: Scattering loss, Confinement loss, Low effective material loss, Single Mode H-PCF, Communication in terahertz optical Fiber.

Posted Date: August 30th, 2021

DOI: https://doi.org/10.21203/rs.3.rs-487498/v1

License: (c) (i) This work is licensed under a Creative Commons Attribution 4.0 International License.

Read Full License 


\title{
Low effective material loss TOPAS based single-mode photonic crystal fiber with high core power fraction in the THz waveguiding
}

\author{
Md. Selim Hossain ${ }^{1}$, Shuvo Sen ${ }^{2 *}$ \\ ${ }^{1}$ Department of Computer Science and Engineering, Khwaja Yunus Ali University, Enayetpur, \\ Sirajganj, Bangladesh \\ ${ }^{2}$ Department of Information and Communication Technology (ICT), Mawlana Bhashani Science \\ and Technology University (MBSTU), Santosh, Tangail-1902, Bangladesh \\ *Corresponding Author: Mawlana Bhashani Science and Technology University, Santosh, \\ Tangail-1902, Bangladesh \\ Email: shuvombstu.it12009@gmail.com
}

\begin{abstract}
In this study, five layers of hexagonal cladding and two elliptical air holes based on photonic crystal fiber are discussed highly for many communication areas by decreasing different types of losses such as effective material loss (EML), scattering loss, and confinement loss in the terahertz (THz) waveguiding. Our suggested fiber (H-PCF) and all simulation results are obtained with the finite element method (FEM) and the perfectly matched layer (PML) boundary conditions based COMSOL Multiphysics software have been used to design in the THz region. After investigating all the graphical results, this optical communication-related H-PCF fiber discloses an extremely low effective material loss (EML) of $0.0184 \mathrm{~cm}^{-1}$, with an effective area of $7.07 \times 10-8 \mathrm{~m}^{2}$ and flow of power in the core region of $88 \%$ at 1 terahertz $(\mathrm{THz})$. Here, other simulation parameters such as confinement loss, scattering loss, and V-parameter are also presented with a proper graph. So, we can easily say that the reported H-PCF fiber is strongly appropriate for different types of short and long-distance communication applications in the terahertz (THz) wave pulse region.
\end{abstract}

Index Terms - Scattering loss, Confinement loss, Low effective material loss, Single Mode H-PCF, Communication in terahertz optical Fiber.

\section{Introduction}

Nowadays, researchers are trying to investigate the high-level research work on wireless communication systems at $\mathrm{THz}$ frequency to increase more capacity compared to the previous system. Terahertz $(\mathrm{THz})$ radiation which changing from 0.1 to $10 \mathrm{THz}$ has gained considerable interest due to its numerous functional uses related to electromagnetic radiation [1-2]. The assortment of $\mathrm{THz}$ frequency exists between the microwave and infrared radiation (IR) in the 
electromagnetic range. The $\mathrm{THz}$ frequency range shows the fascinating development in the field of sensing [3], spectroscopy [4], pharmaceutical drug testing [5], biomedical sensing [6], telecommunications [7], weapons in a non-destructive way [8], DNA Hybridization [9], etc. The leading sources of $\mathrm{THz}$ radiation are high-frequency Gunn diodes, far-infrared (FIR) gas laser, quantum cascade laser (QCL), free-electron laser, etc. Moreover, Schottky barrier diodes, pair braking detectors, hot electron mixers, field-effect transistor detectors, Bolometers, etc. are the fundamental T-ray detector. The necessity of high bandwidth in wireless schemes has been increased due to the unoccupied bandwidth for the current communication scheme. The transmission scheme of $\mathrm{THz}$ frequency largely depends on the free space medium but most of the $\mathrm{THz}$ waveguides experience an unavoidable absorption loss, path loss, difficult integration with other components during free-space propagation [10].

Over the last few years, some metallic and dielectric waveguides for the $\mathrm{THz}$ spectrum have been reported such as Bragg fibers [11], dielectric metal-coated hollow glass tubes [12], bare metal wires [13], parallel-plate waveguides [14], sub-wavelength porous fibers [15], single metallic wires [16], plastic ribbon waveguides [17]. Recently, porous core photonic crystal fibers (PCFs) have obtained significant interest because of their versatility in structural nature and desirable optical guiding properties such as high core power fraction, lower effective material loss, lower dispersion, highly birefringence, high nonlinearity, lower bending loss. Total internal reflection (TIR) and photonic bandgap (PGB) are two basic optical guiding properties are found in PCF. If the light is confined in a higher region of the refractive index in solid-core PCF then the total internal reflection can be optimized. Numerous polymers have been used as background materials in microstructure core PCF to control the optical guiding properties such as TOPAS, Tellurite, Zenox, poly methyl-methacrylate (PMMA), Graphene, Teflon [18-23], etc.

To get a higher sensitivity across the EM spectrum, many standard articles regarding PCFs have already been investigated. Islam et al. [24] anticipated a porous-core spiral shape photonic crystal fiber (PCF). Their proposed model obtained the EML and EA of $0.1 \mathrm{~cm}^{-1}$ and $1.82 \times$ 10-7 m2 accordingly at $1 \mathrm{THz}$ frequency. But their proposed model showed higher EML. In 2016, Hasan et al. [25] explored hexagonal PCFs that gained EML of $0.089 \mathrm{~cm}^{-1}$ at $1 \mathrm{THz}$ frequency. Saiful et al. [26] suggested a rotated hexagonal porous core with circular shape 
cladding and obtained an EML of $0.053 \mathrm{~cm}^{-1}$ with a dispersion of $0.25 \mathrm{ps} / \mathrm{THz} / \mathrm{cm}$. In 2018 , Rana et al. [27] proposed a hexagonal-shaped hole combined within the core of a Kagome lattice PCF. Their proposed model displays EML of $0.029 \mathrm{~cm}^{-1}$ and core power fraction of $33 \%$ at 1.3 $\mathrm{THz}$ frequency. In the same year, Sultana et al. [28] designed a hexagonal shape cladding with elliptical core PCF obtain EML of $0.05 \mathrm{~cm}^{-1}$ and very high birefringence of 0.086 . From the previous background, we have got comparably higher EML and some important aspects such as power fraction (PF) and bending loss were not explored. An elliptical core PCF with kagome lattice cladding where EML of $0.056 \mathrm{~cm}^{-1}$ and lower dispersion of $0.27 \pm 0.18 \mathrm{ps} / \mathrm{THz} / \mathrm{cm} 1 \mathrm{THz}$ operating frequency was anticipated in 2019 by Saiful [29].

In this paper, we have designed a TOPAS based hexagonal shape of PCF with an elliptical core has been introduced in the THz regime. The proposed model shows a low EML of $0.0184 \mathrm{~cm}-1$ with $80 \%$ core power fraction and a large effective area of $7.07 \times 10-8 \mathrm{~m} 2$ at $1 \mathrm{THz}$ optical frequency.

\section{Design Methodology}

The cross views of H-PCF are exposed in Fig. 1. where $\Lambda_{1}$ and $d_{1}$ are defined by the pitch and diameter of our design concept. The constraints $d_{1} / \Lambda_{1}$ is called the air filling ratio and this ratio tries to guard against collapse between two AHs and the background material of TOPAS helps to reduce many types of losses. $\Lambda_{c}, d_{a}$ and $d_{b}$ constraints are called the pitch and diameters of the two elliptical AHs similarly. Here, we find the numerical properties such as effective area, scattering loss, V-parameter, EML, fiber power fraction, and confinement loss of the fiber in the $\mathrm{THz}$ region with the COMSOL Multiphysics software. The optimum constraints are cladding diameter $\mathrm{d}_{1}=\mathrm{d}_{2}=\mathrm{d}_{3}=\mathrm{d}_{4}=\mathrm{d}_{5}=300 \mu \mathrm{m}$, cladding pitch $\Lambda_{1}=\Lambda_{2}=\Lambda_{3}=\Lambda_{4}=\Lambda_{5}=400 \mu \mathrm{m}$, core diameter $\mathrm{d}_{\mathrm{a}}=52 \mu \mathrm{m}, \mathrm{d}_{\mathrm{b}}=167 \mu \mathrm{m}$ and core pitch $\Lambda_{\mathrm{c}}=100 \mu \mathrm{m}$. 


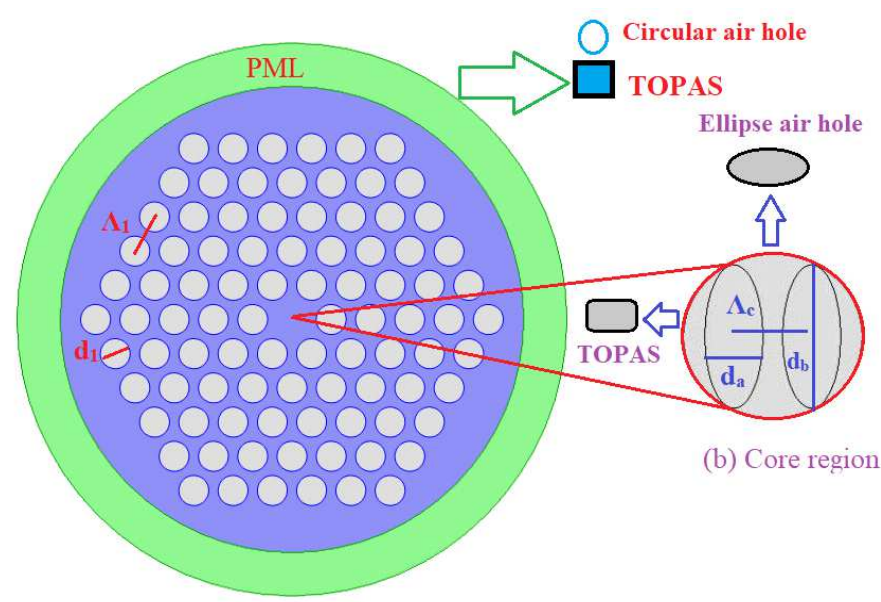

(a) Cladding region

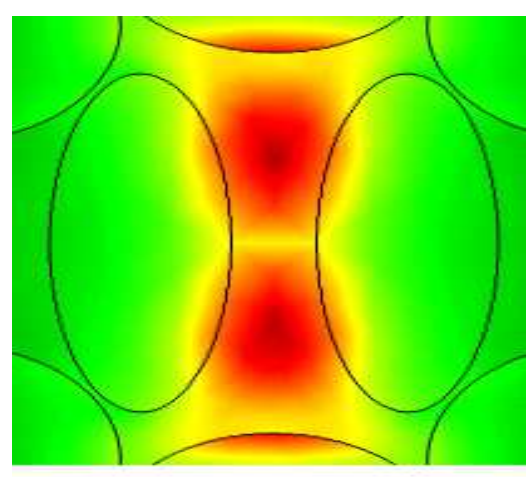

(c) Mode field distribution

Fig. 1: Pictorial views of H-PCF along with (a) Cladding region (b) Core region and (c) Mode field distribution.

\section{Numerical Analysis:}

Background material (TOPAS) has been used of this suggested H-PCF fiber to reduce the effective material loss (EML). Here, EML $\alpha_{\text {eff }}$ is premeditated by [31]:

$$
\alpha_{\text {effective }}=\sqrt{\frac{\varepsilon_{0}}{\mu_{0}}}\left(\frac{\int_{\text {mat }}{ }^{n} \text { mat }|E|^{2} \alpha_{\text {mat }} d A}{\left|\int_{\text {all }} S_{Z} d A\right|}\right)\left(\mathrm{cm}^{-1}\right)
$$

Where, $\alpha_{\text {mat }}$ is the bulk material absorption loss and $n_{\text {mat }}$ is the RI of the material. $\varepsilon_{0}$ is the relative permittivity and the permeability of free space is $\mu_{0} . S_{z}=\frac{1}{2}\left(E \times H^{*}\right)$. $z$ is the pointing vector, where, $\mathrm{z}$ is component of $\mathrm{S}_{\mathrm{z}}, \mathrm{E}$ and $\mathrm{H}^{*}$ are electric field apparatuses and the complex couple of the magnetic field.

SL of H-PCF fiber is thought-out by the subsequent equation [32]: 


$$
\alpha_{\mathrm{R}}=\mathrm{C}_{\mathrm{R}} \times\left(\frac{\mathrm{f}}{\mathrm{c}}\right)^{4}(\mathrm{~dB} / \mathrm{km})
$$

Where, $C_{R}$ is called the scattering coefficient.

The low confinement loss-based PCF fiber is highly used for different types of communication sectors. Here, the confinement loss $\mathrm{L}_{\mathrm{c}}$ is calculted the equation [33]:

$$
\mathrm{L}_{\mathrm{c}}=8.686 \times \mathrm{K}_{0} \operatorname{Im}\left[\mathrm{n}_{\mathrm{eff}}\right](\mathrm{dB} / \mathrm{m})
$$

Where, $K_{0}=\left(\frac{f}{c}\right)$ is the free wave number, $f$ is the frequency and $c$ are the speed of photon. $\operatorname{Im}\left[\mathrm{n}_{\mathrm{eff}}\right]$ is the imaginary part of ERI.

In H-PCF fiber, the principal part is formed by the effective mode area (EMA). Here, the EMA is figured by [34]:

$$
\mathrm{A}_{\text {effective }}=\frac{\left[\int I(r t) r t d r t\right]^{2}}{\left[\int I^{2}(r t) d r t\right]^{2}}
$$

Where, Aeffective is the EMA and $\mathrm{I}(\mathrm{rr})=\left|\mathrm{E}_{\mathrm{rt}}\right|^{2}$ is the cross-sectional electric field intensity.

Power fraction $(\mathrm{PF})$ is resulted by the total power through the H-PCF fiber. So, the PF $\eta$ is intended by [34]:

$$
\eta=\frac{\int_{\mathrm{i}} S_{z} d A}{\int_{\text {all }} S_{z} d A}
$$

$\mathrm{V}$-parameter describes the mode propagation of the H-PCF structure. So, V-parameter is restrained by the following equation [35]:

$$
\mathrm{V}=\frac{2 \pi r f}{c} \sqrt{\mathrm{n}^{2}{ }_{\text {co }}-\mathrm{n}^{2} \mathrm{cl}} \leq 2.045
$$

Where, the core radius is $\mathrm{r}, \mathrm{n}_{\mathrm{co}}$ and $\mathrm{n}_{\mathrm{cl}}$ are signed by the EMI of the core and cladding area.

\section{Analysis of Numerical Results and Discussions:}

COMSOL Multiphysics software has been used to compute entirely optical properties and graphical results from Fig. 2 to Fig. 9 of this the recommended H-PCF are premeditated from 0.8 to $3 \mathrm{THz}$ frequency range. The effective area of the designed PCF is illustrated in figure 2 according to the frequency changing from $1.00 \mathrm{THz}$ to $3.00 \mathrm{THz}$ for $60 \%, 70 \%$, and $80 \%$ porosities. The effective mode area is pragmatic to be decreased gradually that shown in Fig. 2. 
The effective area is computed as $7.07 \times 10^{-8} \mathrm{~m}^{2}, 7.14 \times 10^{-8} \mathrm{~m}^{2}$, and $7.32 \times 10^{-8} \mathrm{~m}^{2}$ for $80 \%, 70 \%$ and $60 \%$ porosities respectively.

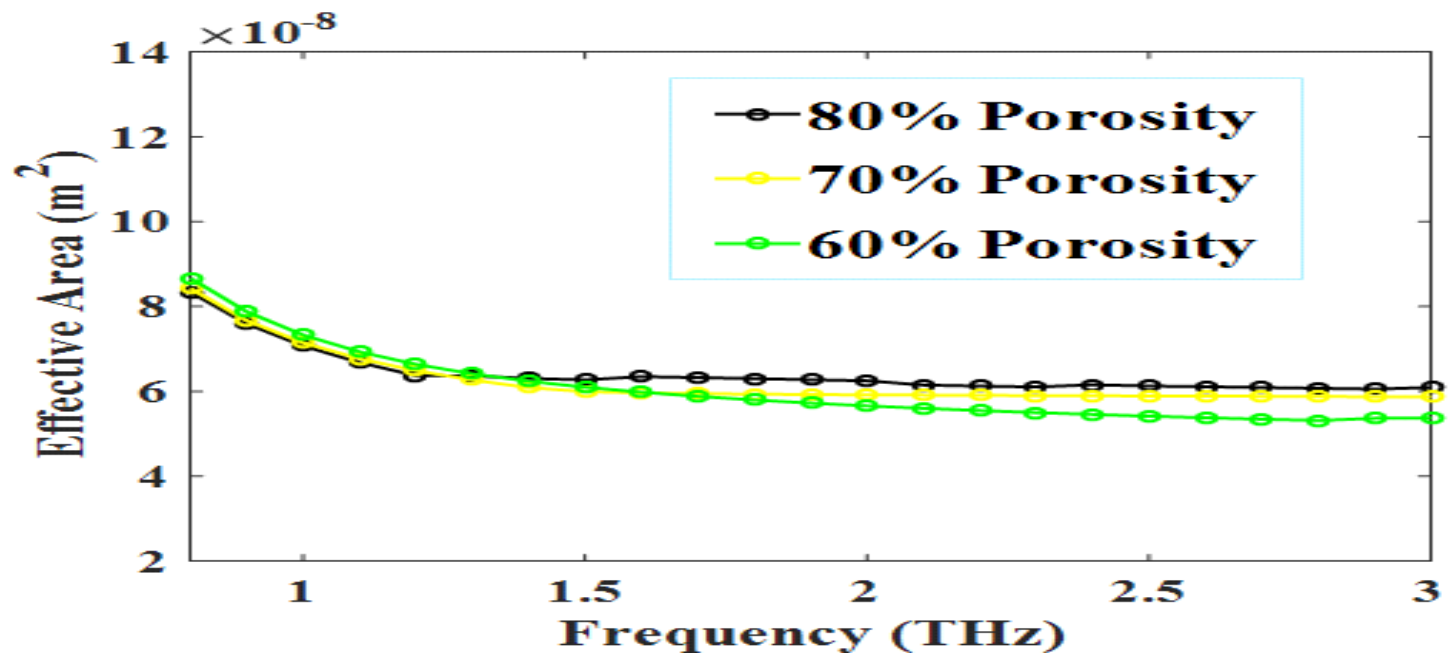

Fig. 2: EA according to the diverse frequencies such as $80 \%, 70 \%$ and $60 \%$ porosities.

Fig. 3 explain the EA in accordance with core diameter $\left(\mathrm{D}_{\text {core }}\right)$ for $60 \%, 70 \%$ and $80 \%$ porosities at $1 \mathrm{THz}$. For optimum core diameter $\mathrm{D}_{\text {core }}=324 \mu \mathrm{m}$, effective area is computed as $8.12 \times 10^{-8} \mathrm{~m}^{2}$ , as $8.38 \times 10^{-8} \mathrm{~m}^{2}$ and as $8.74 \times 10^{-8} \mathrm{~m}^{2}$ for $80 \%, 70 \%$ and $60 \%$ porosities correspondingly for 1 $\mathrm{THz}$ working frequency.

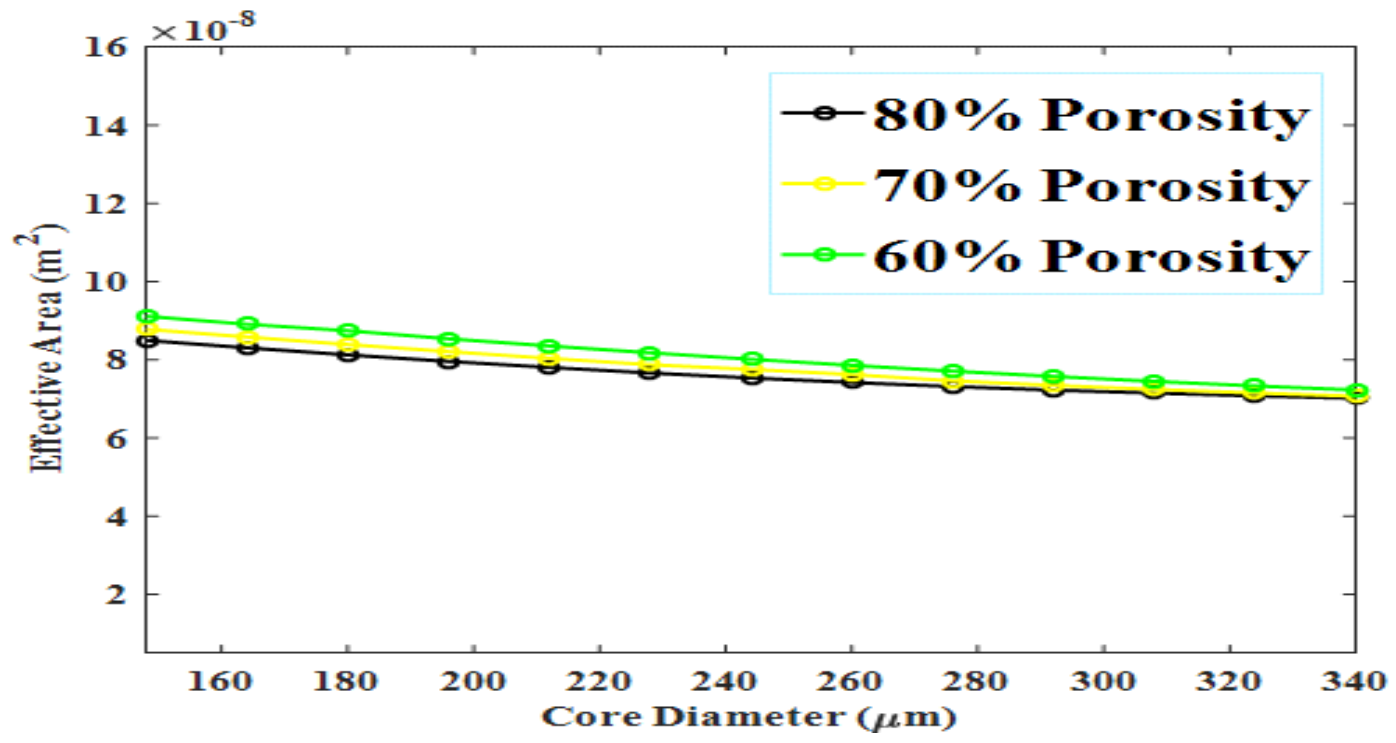

Fig. 3: Effective area according to the different core diameters such as $80 \%, 70 \%$ and $60 \%$ porosities. 
Fig. 4 proves the frequency based effective material loss (EML) graph for several porosities. The figure specifies that the EML of proposed structure decreases with the increasing of frequency over $0.08 \mathrm{THz}$ to $3 \mathrm{THz}$ in electromagnetic spectrum assortment.

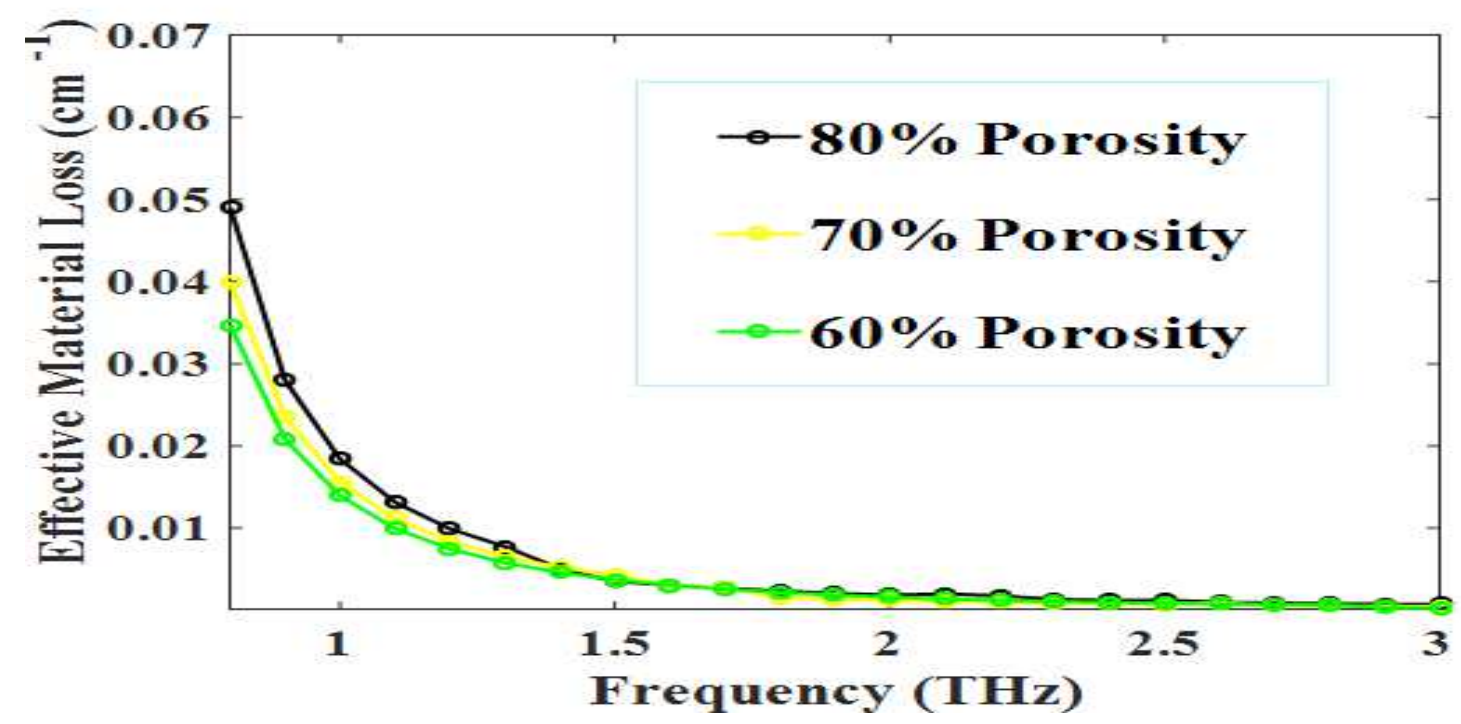

Fig. 4: EML according to the frequency such as $80 \%, 70 \%$ and $60 \%$ porosities.

Therefore, increasing of core porosity of PCF, the generated electromagnetic wave interacts with limited amounts of material; the EML of the proposed fibers then decreases. For optimum design conditions at $1 \mathrm{THz}$ frequency, the EMLs are $0.0184 \mathrm{~cm}^{-1}, 0.0156 \mathrm{~cm}^{-1}$, and $0.0137 \mathrm{~cm}^{-1}$ for $80 \%, 70 \%$ and $60 \%$ porosities correspondingly.

EML due to the changes in core diameter ( $\mathrm{D}_{\text {core }}$ ) of proposed model with $60 \%, 70 \%$ and $80 \%$ porosities have been shown in Fig. 5 at $1 \mathrm{THz}$ frequency. The increases of core diameter the EML is decreasing gradually at optimum design parameter. In our proposed PCFs $\mathrm{D}_{\text {core }}=324$ $\mu \mathrm{m}$ the EML is about $0.0184 \mathrm{~cm}^{-1}$ for $80 \%$ core porosity which is optimum value and not production any complexity in fabrication. At a constant value of $\mathrm{D}_{\text {core }}$, the proposed model shows the different values of EML for different porosities. 


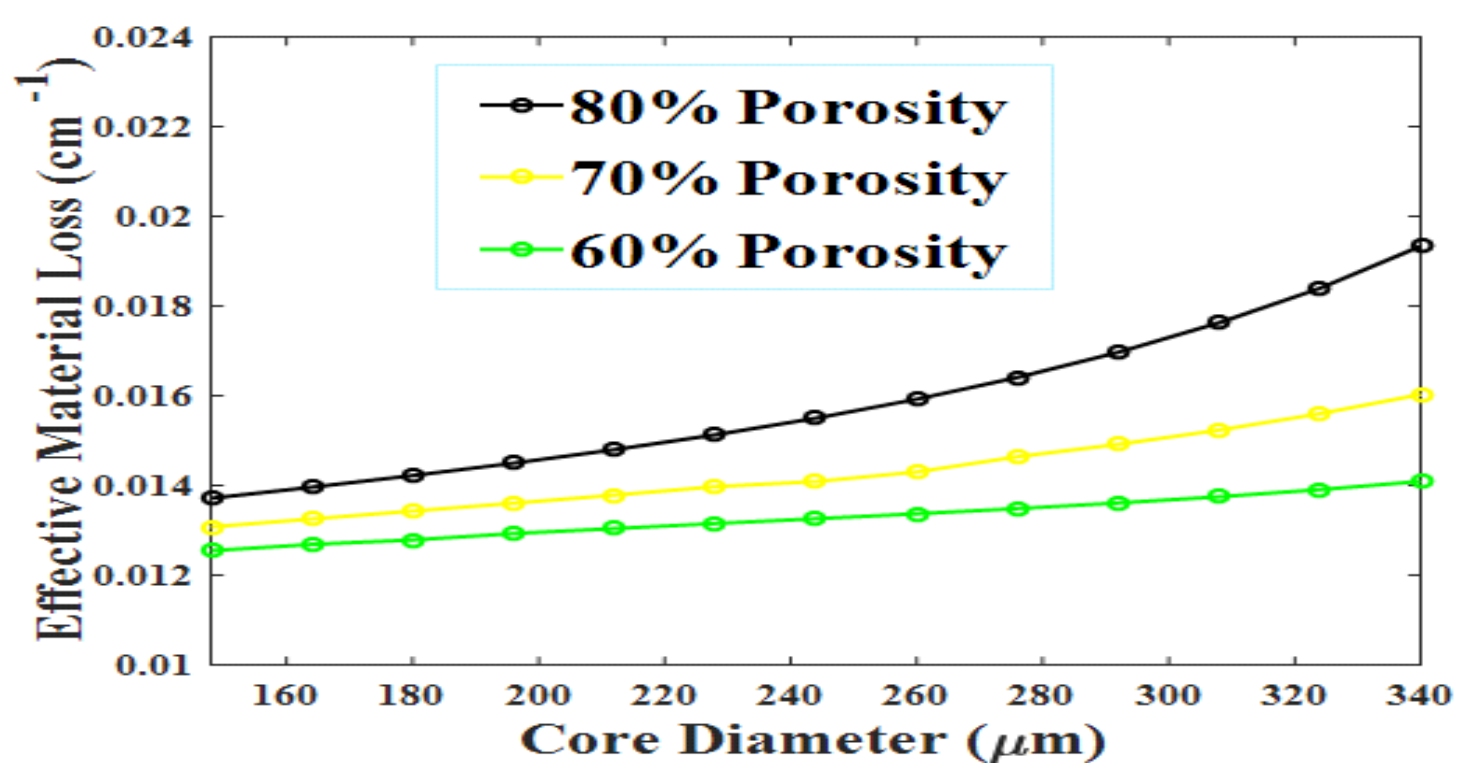

Fig. 5: EML according to the core diameters such as $80 \%, 70 \%$ and $60 \%$ porosities.

Fig. 6 indicates the distribution of power across the core, cladding and materials in accordance with frequency at a fixed $D_{\text {core }}=324 \mu \mathrm{m}$. The experimental frequency varieties within $0.08 \mathrm{THz}$ to $3 \mathrm{THz}$ in electromagnetic spectrum. As it was found that, $80 \%$ optical power generated through the fiber core at frequency $1 \mathrm{THz}$ which means maximum light contact with analytes in the core region. Furthermore, the air holes in cladding region induced light waves to pass within the core and provide maximum core power fraction. The pragmatic power fraction is significantly higher than the previously stated article.

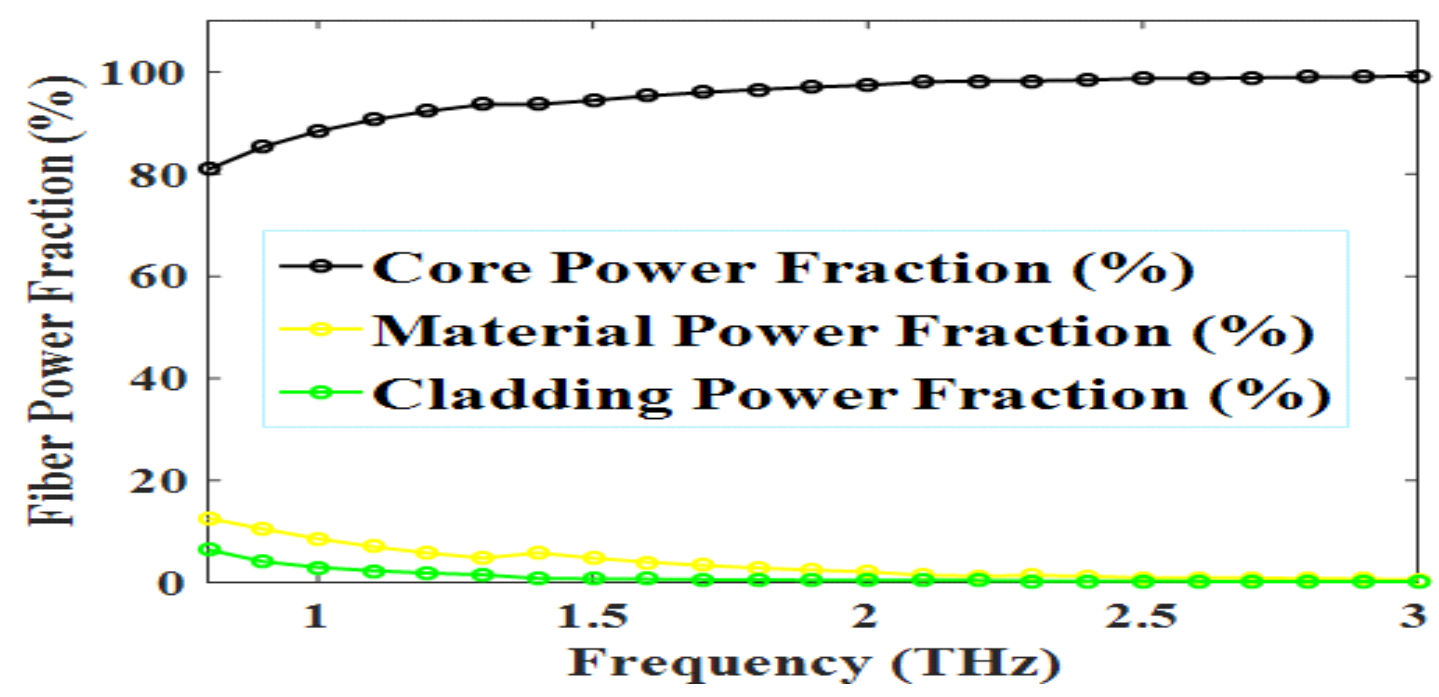

Fig. 6: Power fraction according to the different frequencies for optimum constraints. 
Fig. 7 proves the scattering loss analysis for the variations in wavelength in proposed structure. Scattering loss is an important parameter because it contributes the total losses of the fiber. Scattering loss is increasing with the increases of frequency within 0.08 to $3 \mathrm{THz}$ range appeared in Fig. 7 where's the $D_{\text {core }}=324 \mu \mathrm{m}$. The gained scattering loss of proposed PCF is $1.236 \times 10^{-10}$ $\mathrm{dB} / \mathrm{km}$ at optical wavelength $1 \mathrm{THz}$ which is negligible.

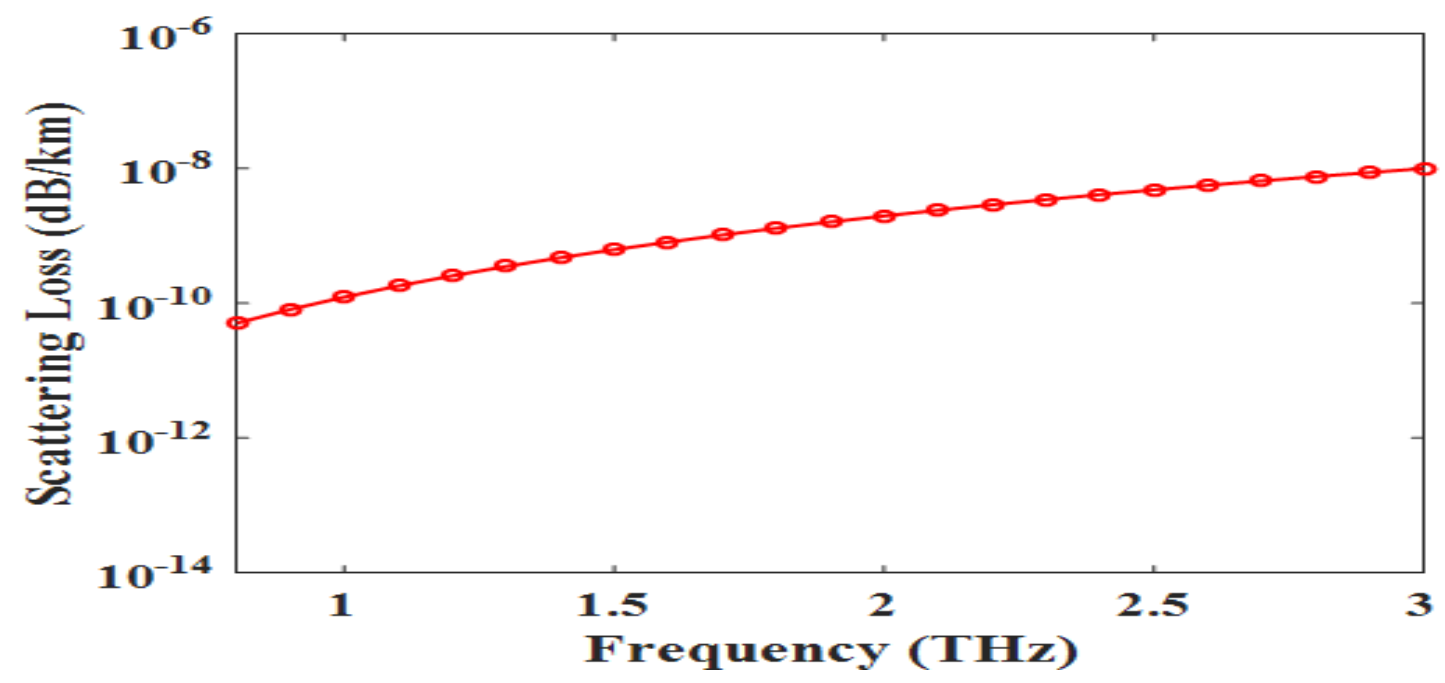

Fig. 7: Scattering loss according to the different frequencies for optimum design parameters.

Fig. 8 illustrates the behavior of CL with respect to frequency at optimum design parameter. Confinement loss (CL) of proposed model is being reduced due to rising of frequency across 0.08 to $3 \mathrm{THz}$ at $\mathrm{D}_{\text {core }}=324 \mu \mathrm{m}$. When light passes through the core with high frequency then it improves the index contrast of core and cladding and thus minimize the confinement loss. It is observed that the confinement loss of proposed structure at optimum design constraints for 1 $\mathrm{THz}$ of the order of $3.36 \times 10^{-15} \mathrm{~dB} / \mathrm{m}$.

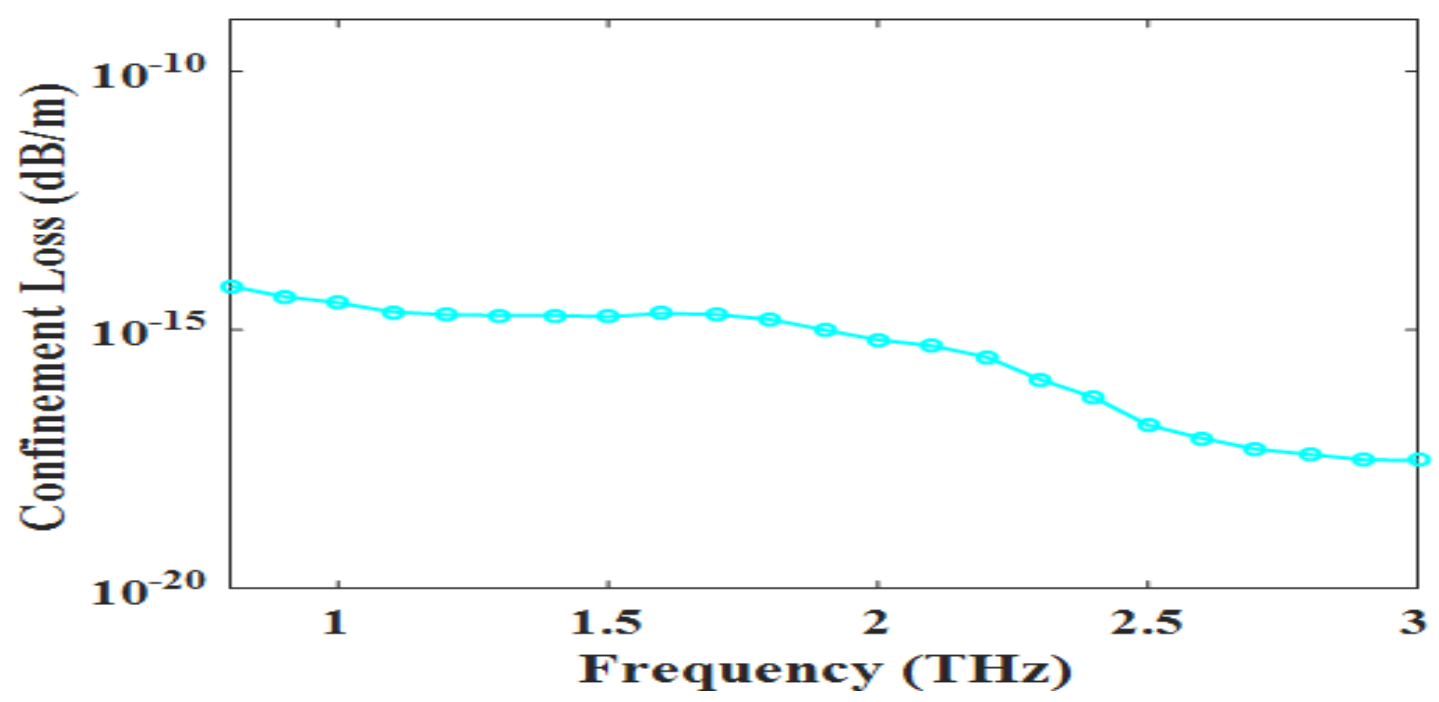


Fig. 8: Confinement loss according to the different frequencies for optimum design parameters.

$V_{\text {eff }}$ is explored as the function of frequency for optimum design constraint at $D_{\text {core }}=416 \mathrm{um}$ which has been revealed in Fig. 9. Her, the optimum constraints are cladding diameter $d_{1}=d_{2}=$ $\mathrm{d}_{3}=\mathrm{d}_{4}=\mathrm{d}_{5}=300 \mu \mathrm{m}$, cladding pitch $\Lambda_{1}=\Lambda_{2}=\Lambda_{3}=\Lambda_{4}=\Lambda_{5}=400 \mu \mathrm{m}$, core diameter $\mathrm{d}_{\mathrm{a}}=52$ $\mu \mathrm{m}, \mathrm{d}_{\mathrm{b}}=167 \mu \mathrm{m}$ and core pitch $\Lambda_{\mathrm{c}}=100 \mu \mathrm{m}$.

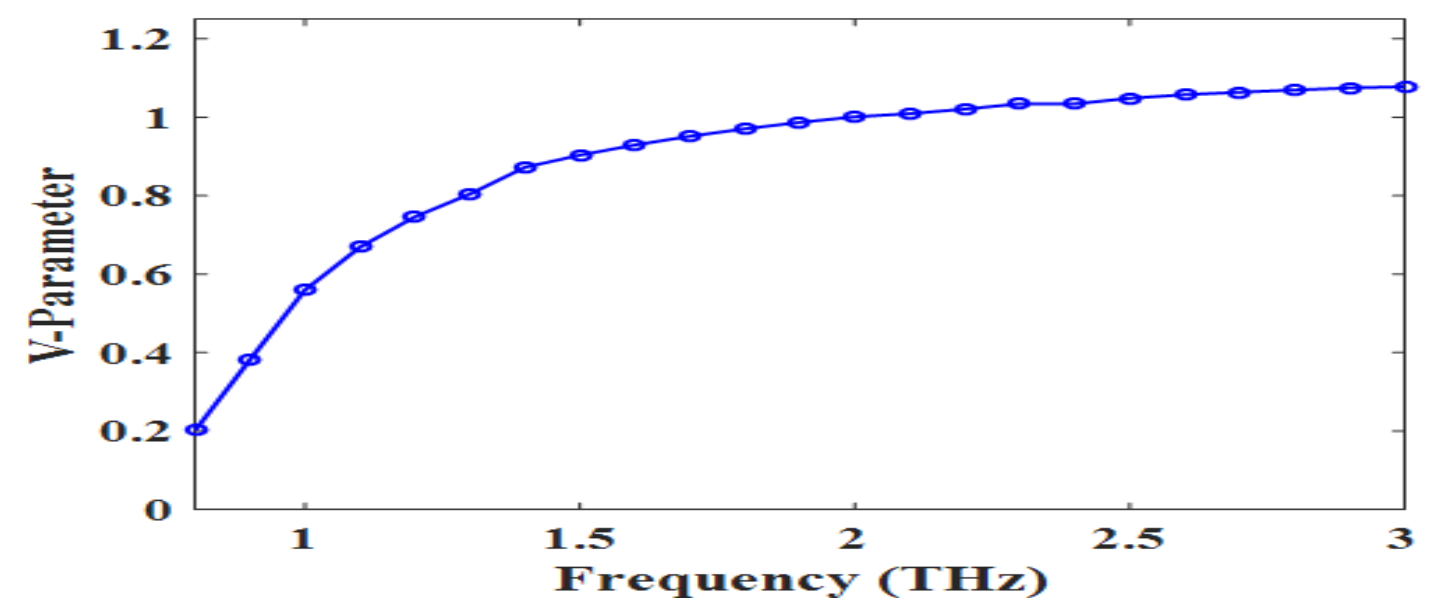

Fig. 9: V-parameter according to the different frequencies for optimum design parameters.

The designed H-PCF shows outstanding EML, confinement loss, core power fraction, and effective area belongings than other designed PCFs at $1 \mathrm{THz}$ functional frequency as providing in Table 1.

Table 1: Propagation comparison among Prior PCFs and of the proposed H-PCF.

\begin{tabular}{cccccc}
\hline Ref. & $\begin{array}{l}\text { EML } \\
\left(\mathrm{cm}^{-1}\right)\end{array}$ & $\begin{array}{c}\text { Porosity } \\
(\%)\end{array}$ & $\begin{array}{l}\text { Power } \\
\text { Fraction }\end{array}$ & $\begin{array}{c}\text { Confinement } \\
\text { Loss }(\mathrm{dB} / \mathrm{m})\end{array}$ & $\begin{array}{l}\text { Effective } \\
\text { Area }\left(\mathrm{A}_{\text {eff }}\right. \\
\left.\left(\mathrm{m}^{2}\right)\right)\end{array}$ \\
\hline$[36]$ & 0.110 & - & - & - & $0.98 \times 10^{-07}$ \\
{$[37]$} & 0.100 & 30 & - & $1.0 \times 10^{-01}$ & $2.3 \times 10^{-07}$ \\
{$[38]$} & 0.1 & - & $32.5 \%$ & - & - \\
{$[39]$} & 0.085 & - & $37 \%$ & - & - \\
{$[40]$} & 0.05 & - & $67.05 \%$ & - & - \\
{$[41]$} & 0.063 & - & $46 \%$ & - & - \\
{$[42]$} & 0.089 & 60 & $37 \%$ & $1.0 \times 10^{-02}$ & $9.77 \times 10^{-08}$ \\
{$[43]$} & 0.076 & 80 & $53 \%$ & $8.96 \times 10^{-01}$ & -
\end{tabular}


$80 \%$

It has been found Table 1 which shows better outputs compare to the former research work. We have found EML $0.007 \mathrm{~cm}^{-1}$, power fraction $80 \%$, Confinement loss $3.37 \times 10^{-14} \mathrm{~dB} / \mathrm{m}$ and effective area is $3.46 \times 10^{-8} \mathrm{~m}^{2}$.

\section{Conclusion:}

An excellent design of five layers hexagonal cladding area based CAHs and two elliptical AHs in the core region are offered for communication applications with decreasing different types of losses such as EML, confinement loss, and scattering loss. TOPAS has been used as background material to remove different losses compare to the previous research work. Moreover, our designed H-PCF structure are designed with the procedure of FEM and PML conditions based on COMSOL Multiphysics software to get the simulated data. The graphical results of this H-PCF fiber show an ultra-low effective material loss (EML) of $0.0184 \mathrm{~cm}^{-1}$, the larger effective area of $7.07 \times 10^{-8} \mathrm{~m}^{2}$, power moving in the core region of $88 \%$, low confinement loss of $3.36 \times 10^{-15}$ $\mathrm{dB} / \mathrm{m}$, and scattering loss of $1.236 \times 10^{-10} \mathrm{~dB} / \mathrm{km}$ respectively at $1 \mathrm{THz}$. So, after investigating all the simulation results, we can say strongly that our H-PCF fiber will be highly appropriate for numerous communication areas in the terahertz $(\mathrm{THz})$ regime.

Conflict of Interest: The authors declare that they have no conflict of interest.

Acknowledgement: The authors are grateful to the participants who contributed to this research. The authors have not received any funding for this research.

\section{References:}

[1] Su-jie Guo, Cun-jun Ruan, De-yin Kong, Jun Dai, Yan Zhang, Wen-long He, "Research on terahertz transmission characteristics of nonpolar liquid based on frequency-domain spectroscopy", Journal of the Optical Society of America B 37:7, pages 1942, 2020

[2] Taherzadeh, M.J.; Karimi, K. "Pretreatment of Lignocellulosic Wastes to Improve Ethanol and Biogas Production: A Review”, Int. J. Mol. Sci. 2008, 9, 1621-1651.

[3] D. G. J.Q. Zhang. Waveguide terahertz time-domain spectroscopy of nanometer water layers. Optics Letters, 29(14), 1617-1619,2004 
[4] F. A. Mou, M. M. Rahman, M. A. Al Mahmud, M. R. Islam and M. I. H. Bhuiyan, "Design and Characterization of a Low Loss Polarization Maintaining Photonic Crystal Fiber for THz Regime," 2019 IEEE International Conference on Telecommunications and Photonics (ICTP), Dhaka, Bangladesh, 2019, pp. 1-4, doi: 10.1109/ICTP48844.2019.9041715.

[5] Sohel Rana, Sharafat Ali, Nasim Ahmed, Raonaqul Islam, Syed Alwee Aljunid, "Ultra-high birefringent and dispersion-flattened low loss single-mode terahertz wave guiding", Communications IET, vol. 10, no. 13, pp. 1579-1583, 2016.

[6] Raonaqul Islam, G. K. M. Hasanuzzaman, Md. Anwar Sadath, Sohel Rana, Md. Selim Habib, "Extremely low-loss single-mode photonic crystal fiber in the terahertz regime", Electrical \& Electronic Engineering (ICEEE) 2015 International Conference on, pp. 289-292, 2015.

[7] B.M.A. Rahman, M. Uthman, A. Quadir, K. T. V. Grattan, C. Markides, C. Themistos, "Emergence of THz technologies and design and optimisation low-loss waveguides and devices", Electrical and Computer Engineering (ICECE) 2014 International Conference on, pp. 389-393, 2014.

[8] P. H. B. M. Nagel, M. Brucherseifer, H. Kurz, A. Bosserhoff, R. Bttner. Integrated THz technology for label-free genetic diagnostics. Applied Physics Letters, 80(2002) 154-156.

[9] Ahmed, K., Islam, M., Sen, S., Paul, B.K., Chowdhury, S., Hasan, M., Uddin, M.S., Asaduzzaman, S. and Bahar, A.N., 2017. Low-loss single mode terahertz microstructure fiber with near-zero-flattened dispersion. Advanced Science, Engineering and Medicine, 9(10), pp.829-836.

[10] Md. Ahasan Habib, Md. Shamim Anower, "A novel low loss porous-core photonic crystal fiber for terahertz wave transmission", Electrical Computer and Communication Engineering (ECCE) International Conference on, pp. 56-59, 2017.

[11] J. Sultana, M.S Islam, J. Atai, M.R. Islam and D. Abbott, "Near-zero dispersion flattened low-loss porouscore waveguide design for terahertz signal transmission", Optical Engineering, vol. 56, pp. 076114, 2017.

[12] Md. Rabiul Hasan, Sanjida Akter, Sohel Rana, Sharafat Ali, "Hybrid porous-core microstructure terahertz fibre with ultra-low bending loss and low effective material loss", Communications IET, vol. 12, no. 1, pp. 109-113, 2018.

[13] K. Tajima, K. Nakajima, K. Kurokawa, N. Yoshizawa and M. Ohashi, "Low-loss photonic crystal fibers," Optical Fiber Communication Conference and Exhibit, Anaheim, CA, 2002, pp. 523-524, doi: 10.1109/OFC.2002.1036529.

[14] X. Yang, and P. Chahal, "THz ribbon waveguides using polymer-ceramic nanocomposites," In Proc. 62nd Electronic Components and Technology Conference (ECTC), pp.225-230, May 29 -June 1, 2012

[15] Z.D. Taylor, R.S. Singh, D.B. Bennett, P. Tewari, C.P. Kealey, N. Bajwa, M.O. Culjat, A. Stojadinovic, H. Lee, J. Hubschman, E.R. Brown, and W.S. Grundfest, "THz Medical Imaging: in vivo Hydration Sensing", Terahertz Science and Technology, vol.1, no.1, pp. 201-219, Sep, 2011. 
[16] M. S. Islam, J. Sultana, S. Rana, M. R. Islam, M. Faisal, S. F. Kaijage, and D. Abbott, Optical Fiber Technology 34, 6 (2017)

[17] K. Tajima, K. Nakajima, K. Kurokawa, N. Yoshizawa and M. Ohashi, "Low-loss photonic crystal fibers," Optical Fiber Communication Conference and Exhibit, Anaheim, CA, 2002, pp. 523-524, doi: 10.1109/OFC.2002.1036529.

[18] X. Tang, Y. Jiang, B. Sun, J. Chen, X. Zhu, P. Zhou, D. Wu, and Y. Shi, IEEE Photonics Technology Letters 25, 331 (2013)

[19] G. Pastorelli, T. Trafela, P. F. Taday, A. Portieri, D. Lowe, K. Fukunaga, and M. Strlic, Analytical and Bioanalytical Chemistry 403, 1405 (2012)

[20] Dash, J.N., Jha, R.: Graphene-based birefringent photonic crystal fber sensor using surface plasmon resonance. IEEE Photon. Technol. Lett. 26(11), 1092-1095 (2014)

[21] M. Goto, A. Quema, H. Takahashi, S. Ono, and N. Sarukura, Japanese Journal of Applied Physics 43, 317 (2004)

[22] R. Islam, S. Rana, R. Ahmad and S. F. Kaijage, "Bend-Insensitive and Low-Loss Porous Core Spiral Terahertz Fiber," in IEEE Photonics Technology Letters, vol. 27, no. 21, pp. 2242-2245, 1 Nov.1, 2015, doi: 10.1109/LPT.2015.2457941

[23] Hasan, M.R.; Islam, M.A.; Anower, M.S.; Razzak, S.M.A. Low-loss and bend-insensitive terahertz fiber using a rhombic-shaped core. Appl. Opt. 2016, 55, 8441-8447

[24] Islam, M.S., Rana, S., Islam, M.R., Faisal, M., Rahman, H., Sultana, J.: Porous core photonic crystal fiber for ultra-low material loss in THz regime. IET Commun. 10(16), 2179-2183 (2016)

[25] Rana, S., Rakin, A. S., Hasan, M. R., Reza, M. S., Leonhardt, R., Abbott, D., \& Subbaraman, H. (2018), "Low loss and flat dispersion Kagome photonic crystal fiber in the terahertz regime". Optics Communications, 410, 452-456.

[26] Sultana, J., Islam, M.S., Faisal, M., Islam, M.R., Ng, B.W.H., Ebendorf-Heidepriem, H., Abbott, D.: Highly birefringent elliptical core photonic crystal fber for terahertz application. Opt. Commun.407, 92-96 (2018)

[27] M. S. Islam, M. Faisal, S. M. A. Razzak, "Dispersion flattened extremely high-birefringent kagome lattice elliptic core photonic crystal fiber in THz regime,” Optical and Quantum Electronics, 51:35, (2019)

[28] B. K. Paul, K. Ahmed, "Analysis of terahertz waveguide properties of Q-PCF based on FEM scheme," Optical Materials 100, 109634, (2020)

[29] Hossain, M.S., Sen, S. "Design and Performance Improvement of Optical Chemical Sensor Based Photonic Crystal Fiber (PCF) in the Terahertz (THz) Wave Propagation", Silicon (2020), https://doi.org/10.1007/s12633-020-00696-8

[30] Liang, J., Ren, L., Chen, N., \& Zhou, C. (2013). Broadband, low-loss, dispersion flattened porous-core photonic bandgap fiber for terahertz (THz)-wave propagation. Optics Communications, 295, 257-261.

[31] Francesco Poletti, Marco Petrovich and David Richardson, "Hollow-core photonic bandgap fibers: Technology and applications", Nano-photonics, vol. 2, no. 10, 2013. 
[32] I.K. Yakasai, P.E. Abas, H. Suhaimi, F. Begum, "Low loss and highly birefringent photonic crystal fiber for terahertz applications”, Optik, 206 (April 2020), p. 1643221

[33] Nagel, M., Haring Bolivar, P., Brucherseifer Bosserhoff, A., \& Büttner, R. (2002). Integrated THz technology for label-free genetic diagnostics. Applied Physics Letters, 80(1), 154-156., M., Kurz, H.,

[34] K. Tajima, "Low loss PCF by reduction of hole surface imperfection," 33rd European Conference and Exhibition of Optical Communication - Post-Deadline Papers (published 2008), Berlin, Germany, 2007, pp. $1-2$.

[35] Islam R, Rana S, Ahmad R, Kaijage SF. Bend-insensitive andlow-loss porous core spiral terahertz fiber. IEEE PhotonTechnol Lett 2015; 27(21):2242-2245. DOI:10.1109/LPT.2015.2457941.

[36] G.K.M. Hasanuzzaman, S. Rana, M.S. Habib, A novel low loss, highly birefringent photonic crystal Fiber in THz regime, IEEE Photonics Technol. Lett. 28 (April 8) (2016) 899-902.

[37] M.R. Hasan, M.S. Anower, M.A. Islam, S.M.A. Razzak, Polarization-maintaining low-loss porous-core spiral photonic crystal fiber for terahertz wave guidance, Appl. Opt. 55 (15) (2016) 4145-4152.

[38] Takashi Matsui, Taiji Sakamoto, Kyozo Tsujikawa, Shigeru Tomita, Makoto Tsubokawa, "Single-Mode Photonic Crystal Fiber Design with Ultra large Effective Area and Low Bending Loss for Ultrahigh-Speed WDM Transmission", Lightwave Technology Journal of, vol. 29, no. 4, pp. 511-515, 2011.

[39] M.S. Islam, et al., Zeonex-based asymmetrical terahertz photonic crystal fiber for multichannel communication and polarization maintaining applications, Appl. Opt. 57 (4) (2018) 666.

[40] Hasan MR, Islam MA, Anower MS, Razzak SM. Low-loss and bend-insensitive terahertz fiber using a rhombic-shaped core.Appl Opt 2016;55(30):8441-8447. DOI:10.1364/AO.55.008441.

[41] Hasan MR, Islam MA, Rifat AA. A single mode porous-core square lattice photonic crystal fiber for $\mathrm{THz}$ wave propagation.J Eur Opt Soc Rapid Publ 2016;12(1):15. DOI:10. 1186/s41476-016-0017-5.

[42] Ahmed K, Chowdhury S, Paul BK, Islam MS, Sen S, Islam MI,et al. Ultrahigh birefringence, ultralow material loss porous core single-mode fiber for terahertz wave guidance. Appl Opt 2017;56(12):34773483. DOI:10.1364/AO.56.003477.

[43] Rana S, Hasanuzzaman GK, Habib S, Kaijage SF, Islam R.Proposal for a low loss porous core octagonal photonic crystal fiber for T-ray wave guiding.Opt Eng 2014;53(11):115107-115107. DOI:10.1117/1.OE.53.11.115107.

[44] Saiful Islam Md, Sultana J, Ahmed K, Rakibul Islam M, Dinovitser A, Wai-Him Ng B and Abbott D, 2018.A novel approach for spectroscopic chemical identification using photonic crystal fiber in the terahertz regime. IEEE Sens. J. 18: 575-582.

[45] Saiful Islam Md, Sultana J, Rifat A A, Dinovitser A, Wai-Him Ng B and Abbott D, 2018.Terahertz sensing in a hollow core photonic crystal fiber. IEEE Sens. J. 18: 4073-4080 